Revue internationale P.M.E.

\title{
GRP Lab : d'une théorie du business model à la réalisation d'une plateforme numérique de sensibilisation, formation et accompagnement à l'entrepreneuriat
}

\section{Thierry Verstraete}

Volume 28, numéro 3-4, 2015

URI : https://id.erudit.org/iderudit/1035408ar

DOI : https://doi.org/10.7202/1035408ar

Aller au sommaire du numéro

Éditeur(s)

Editions EMS - In Quarto SARL

ISSN

0776-5436 (imprimé)

1918-9699 (numérique)

Découvrir la revue

Citer ce document

Verstraete, T. (2015). GRP Lab : d'une théorie du business model à la réalisation d'une plateforme numérique de sensibilisation, formation et accompagnement à l'entrepreneuriat. Revue internationale P.M.E., 28(3-4), 17-26.

https://doi.org/10.7202/1035408ar 


\title{
GRP Lab : d'une theorie du business model à la réalisation d'une plateforme numérique de sensibilisation, formation et accompagnement à l'entrepreneuriat
}

\author{
Thierry VERSTRAETE \\ Professeur à l'Institut d'Administration des Entreprises de l'Université de Bordeaux
}

Les positions pragmatistes en recherche relèvent souvent de l'intention, se déclarent parfois, mais se manifestent plus rarement... «GRP Lab » répond au souhait d'une équipe de recherche de voir ses travaux se décliner en pratique. Il se matérialise par le site http://grp-lab.com. Cette forme numérique, gratuitement accessible, est le produit de la valorisation d'une recherche sur le business model (BM dans la suite du texte) ayant consisté à en comprendre l'origine, le bon sens, la nature, les composantes et les fonctions ${ }^{1}$.

GRP Lab est défini comme une plateforme de compétences dédiée à la sensibilisation, à la formation et à l'accompagnement à l'entrepreneuriat. Il se base sur le modèle GRP. Le modèle GRP définit le BM comme une convention (éclairage conceptuel de la notion de BM par la théorie des conventions) relative à la génération $(G)$ de la valeur, à la rémunération $(R)$ de la valeur et au partage (P) de la valeur (Verstraete et Jouison-Laffitte, 2009 ; 2011a et 2011b). Ses utilisateurs parlent de BM GRP. La vulgarisation de la théorie lors de son transfert sur GRP Lab a traduit le mot « convention » par « représentation partagée ».

Les outils proposés par GRP Lab sont à l'usage de l'encadrant et de l'encadré, puisqu'il ne s'agit surtout pas de court-circuiter la relation d'accompagnement. Celle-ci participe à la réussite des projets. L'encadrant peut être un conseiller en création d'entreprise, un formateur en entrepreneuriat, un consultant. L'encadré peut être le créateur d'une entreprise concevant son affaire, l'étudiant suivant un séminaire de sensibilisation ou une formation en entrepreneuriat, l'entrepreneur d'une entreprise établie ou encore le responsable d'une organisation à but non lucratif. Ces acteurs ont fait l'objet de plusieurs études de cas ou de recherches-actions. Rien n'est anecdotique sur le site de GRP Lab ; ce qu'il propose est le résultat de recherches. C'est cette activité scientifique qui a été reconnue dans le cadre d'un appel IdEx.

En effet, GRP Lab a été labellisé centre d'innovation sociétale (CIS) de l'initiative d'excellence (IdEx) de l'Université de Bordeaux². Dans ce cadre, GRP Lab a bénéficié d'un inves-

\footnotetext{
1 L'expression «business model " s'est installée dans le monde de la pratique auquel s'intéresse cette rubrique. Parfois traduite "modèle d'affaires » ou "modèle économique », le résultat n'est pas satisfaisant (surtout la seconde qui n'est qu'une partie d'un business model).
}

2 L'action IdEx du programme des investissements d'avenir de l'État français vise à soutenir et à 
tissement principalement dirigé vers le développement de la plateforme en ligne ${ }^{3}$. La Chaire Entrepreneuriat de la fondation Bordeaux université soutient également le projet. Démarré en 2012, GRP Lab a été inauguré en mars 2014. Il valorise une somme de travail dont les premiers jalons remontent à une quinzaine d'années, mais il transfère principalement les connaissances apportées par l'équipe Entrepreneuriat de l'IRGO (Institut de recherche en gestion des organisations) de l'Université de Bordeaux. Celle-ci travaille 4 axes : BM et pédagogie de l'entrepreneuriat, BM et accompagnement de projets (création, reprise, développement, évaluation...), BM et entrepreneuriat social, BM dans le secteur du vin (important pour la France, notamment pour la région de Bordeaux) ${ }^{4}$.

La suite de cette note rappelle le problème de recherche initial et ses évolutions (1), présente les méthodes utilisées pour approcher les terrains (2), les enseignements que les parties en ont tirés en prenant quelques exemples de recherches réalisées ou en cours avant de conclure. GRP Lab insistant sur le "storytelling », la suite du texte prend volontairement une forme narrative, et chronologique, pour exposer à la fois la genèse et la construction du projet. La première façon de communiquer, n'est-ce pas de raconter?

\section{DE LA GENĖSE DU MODĖLE Ȧ LA CRÉATION DE GRP LAB : COMPRENDRE, AGIR ET VALORISER}

En 1999, alors que j'avais monté des cours en entrepreneuriat à l'Université, un collègue féru de nouvelles technologies demande si je parle de BM aux étudiants. Question embarrassante car la réponse est négative. À l'époque, les cours en entrepreneuriat s'appuient sur le plan d'affaires.

Si j'avais entendu ou lu à quelques reprises l'expression «BM ", je n'en avais qu'une vague idée. Mais l'embarras était surtout lié au fait qu’un porteur de projet formé pouvait se trouver face à un partenaire l'interrogeant sur son BM sans savoir le distinguer du plan d'affaires (l'utilisation de la forme anglaise "business plan " ajoutant au risque d'amalgame). On ne sera pas certain qu'aujourd'hui tous les acteurs de l'entrepreneuriat fassent la différence...

promouvoir, au niveau international, les établissements reconnus pour leur excellence scientifique, sur la base de projets en relation avec le milieu socioéconomique et structurés autour de compétences. Dans ce contexte, les CIS de l'IdEx Bordeaux ont la mission d'offrir au milieu socioéconomique des compétences construites par les centres de recherche de l'Université. Sur le campus bordelais, ils sont aujourd'hui au nombre de trois : le forum Montesquieu dans la discipline du droit, l'outil d'intelligence technologique Via-Inno porté par une équipe d'économistes et GRP Lab dans le domaine de l'entrepreneuriat.

3 Hélène Jacquet, à la direction de la MIA (Mission Investissement d'avenir), a accompagné l'équipe dans le montage du dossier. Elle avait préalablement apporté son soutien lors des actions menées en faveur de l'entrepreneuriat étudiant et connaissait les compétences de l'équipe.

4 Il est tout à fait possible que des recherches, plus ponctuelles à ce jour, s'inscrivent dans un autre thème. Je pense notamment à une recherche en cours sur les BM dans le domaine culturel, notamment depuis le recrutement récent de Federica Antonaglia, et à un travail singulier aux e-BM dans lequel s'implique notre ingénieur de recherche Vincent Blazquez. 
Il fallait, à l'enseignant, endosser son rôle de chercheur pour tirer cela au clair... Le premier essai a été publié en 2000 sous la forme d'un chapitre d'ouvrage pour lequel, avec ce collègue taquin, nous avons combiné nos centres d'intérêt (Benavent et Verstraete, 2000) ${ }^{5}$. Le titre de ce chapitre comporte les mots « construction et régénération » qu’on transformerait sans doute actuellement en « design et redesign » (termes très utilisés aujourd'hui).

Avec Bertrand Saporta, dans un ouvrage sur l'entrepreneuriat (Verstraete et Saporta, 2006) nous consacrons une section au BM dont l'un des titres s'intitule «Valeurs, conventions et parties prenantes ». Il intègre les bases d'une conceptualisation non achevée. Le BM est considéré comme un registre conventionnel devant réunir des possesseurs de ressources dont l'engagement suppose qu'ils perçoivent la valeur de l'offre d'une entité capable de la produire, le mode de rémunération de la valeur échangée sur les marchés et ce que nous qualifions de partage du "gâteau ", qui correspond à une répartition de la valeur construite ensemble (entre parties prenantes ; voir les pages 377-378). On est proche du modèle GRP, mais celui-ci n'est pas encore formalisé et, surtout, cette conception ne s'appuie pas sur un état de l'art qui sera réalisé par une collaboration stimulante et pérenne avec Estèle Jouison-Laffitte.

Estèle Jouison-Laffitte était alors depuis peu inscrite en thèse. Respectant la position pragmatiste de l'équipe de recherche en entrepreneuriat de l'IRGO (créée en 2005), le cadre opératoire déployé est une recherche-action sur le terrain de 6 projets de création d'entreprise. L'un des principaux enseignements tiré de ce travail doctoral est que le BM s'avère particulièrement pertinent pour accompagner les porteurs d'un projet de création d'entreprise (Jouison, 2008).

Dans le même temps, nous précisions ensemble les contours du modèle GRP pour le présenter formellement en 2009 dans un ouvrage de langue française, en 2011 dans un article publié dans la revue Management international et dans un ouvrage de langue anglaise la même année (Verstraete et Jouison-Laffitte, 2009 ; 2011a et 2011b). Quelques communications avaient, préalablement et dès 2006, présenté notre conception et un article paru dans la Revue française de gestion avait également participé au souhait de formalisation (Jouison et Verstraete, 2008).

Chemin faisant, tous les membres de l'équipe de recherche en entrepreneuriat de l'IRGO rejoignent les auteurs pour préciser davantage le modèle, réaliser des recherches-actions afin de le décliner de façon pertinente dans les enseignements et dans les protocoles d'accompagnement. À ces travaux scientifiques, dont 6 thèses de doctorat mobilisant le BM GRP ${ }^{7}$, et pédagogiques, s'ajoute la valorisation pratique du modèle avec la naissance de GRP Lab.

5 Benavent, C. et Verstraete, T. (2000). Entrepreneuriat et NTIC - La construction du business model. Dans T. Verstraete (dir.), Histoire d'entreprendre - Les réalités de l'entrepreneuriat. Cormellesle-Royal, EMS.

6 Verstraete, T. et Saporta, B. (2006). Création d'entreprise et entrepreneuriat. Éditions de l'ADREG.

7 La bibliographie intègre les thèses soutenues par des membres de l'équipe ayant utilisé le BM GRP pour mettre au jour les BM des cas étudiés et les analyser afin de servir l'étude de l'objet de la recherche (Servantie, 2010 ; Bousquet, 2014 ; Fouda, 2014) ou ayant placé le BM dans l'objet d'étude (Jouison, 2008 ; Masamba, 2013 ; Meiar, 2015). 
GRP Lab comporte plusieurs rubriques accessibles sur le site http://grp-lab.com : logiciel, cas, équipe, recherche, blogue, enseignement, publication. Parmi les développements numériques mis à disposition de l'internaute, la Web application GRP Storyteller invite le créateur d'entreprise, guidé par son conseiller, à raconter son BM. Elle est gratuite et collaborative, c'est-à-dire que plusieurs membres d'une équipe peuvent travailler sur le même document, en même temps, chacun à partir de son ordinateur ou de sa tablette. Les données sont confidentielles, seuls les invités par le créateur du projet peuvent consulter, ou amender lorsqu'ils y sont autorisés, le contenu du projet. Celui-ci peut être édité pour un envoi par voie électronique ou imprimé pour un transfert physique.

Outre des tutoriels, le site reçoit également des vidéos pédagogiques sur le BM. Elles sont d'une durée d'environ 5 minutes pour servir de base à une discussion que le formateur aura avec les personnes qu'il encadre ${ }^{8}$.

\section{DES ÉTUDES DE CAS ET DES RECHERCHES-ACTIONS POUR APPRÉCIER DIFFÉRENTS CONTEXTES D'UTILISATION DU BUSINESS MODEL GRP}

Les cadres opératoires, conformément à la position pragmatiste de l'équipe, s'appuient sur des études de cas et des recherches-actions. Ces deux types de protocole, pouvant parfaitement s'emboîter, constituent de remarquables occasions de rapprochement avec les terrains. Il s'avère que l'équipe possède une experte pour chacune de ces méthodes (Hlady-Rispal, 2002 ; Jouison-Laffitte, 2009) ayant transmis son savoir aux autres membres.

Contrairement à une idée trop souvent reçue, les terrains sont très intéressés par un regard scientifique. Encore faut-il déployer des protocoles dont ils apprécient l'apport, sans qu'ils aient besoin d'en connaître les soubassements épistémologiques ou les détails méthodologiques. Ceci dit, chacune des recherches conduites par l'équipe prévoit une réunion avec les deux parties (chercheurs et terrain) lors de laquelle les engagements sont exposés (notamment en termes de disponibilité) et les limites sont précisées (par exemple le chercheur n'est pas un consultant). Les études de cas et les recherches-actions provoquent une forte proximité des acteurs, voire une intimité révélant l'âme du contexte investi.

Les recherches de GRP Lab déploient en premier lieu plutôt des études de cas (Hlady-Rispal, 2002) afin de bien comprendre les situations de gestion. Encore récemment, la thèse de Meiar (2015), mobilisant le BM GRP dans un contexte de transmission d'entreprise, a permis d'appréhender ce contexte nouveau pour l'équipe (une recherche-action suivra).

$\mathrm{Au}$ sein des recherches conduites par les membres de GRP Lab, une étude de cas sert la première étape d'identification d'un problème d'une recherche-action pouvant comporter plusieurs boucles, comme le proposent Susman et Evered (1978) ${ }^{9}$. Ces auteurs, dans un texte

8 Ces vidéos sont à cette adresse : http://grp-lab.com/category/videos et toutes les autres sont placées ici : https://vimeo.com/grplab.

9 Susman, G.I. et Evered, R.D. (1978). An assessment of scientific merits of action research. Administrative Science Quaterly, 23(4), 582-603. 
restant d'actualité, considèrent que les sciences de l'organisation vivent une crise dont l'un des symptômes est une sophistication croissante de méthodes inutilisables par les praticiens $^{10}$. Ces méthodes accumulent une connaissance sans que les acteurs censés être intéressés ne puissent la mobiliser, notamment parce que la construction du savoir est trop souvent déconnectée des problèmes rencontrés par ces praticiens. Susman et Evered montrent qu’il s'agit d'une lacune que d'autres perspectives méthodologiques peuvent combler, sous réserve de ne pas verser dans le modèle unique de la science, donc en révisant certains critères de scientificité (par exemple l'idée de « test »). Sans rejeter quelque méthode que ce soit, il n'est pas déraisonnable de considérer qu'en sciences de gestion certains problèmes nécessitent une définition commune (entre chercheurs et acteurs du terrain étudié) pour que le pilotage de l'organisation puisse utiliser la connaissance apportée par des chercheurs qui, par ce principe, peuvent difficilement rester neutres. Susman et Evered proposent la « recherche-action » comme une alternative permettant à l'activité de recherche de rester en phase avec les problèmes auxquels les praticiens font face. Elle nous semble être un cadre opératoire répondant particulièrement bien à la position épistémologique de GRP Lab : produire de la théorie utile.

\section{LES TERRAINS INVESTIS PAR L'ÉQUIPE DE GRP LAB}

Les membres de l'équipe sont, pour la plupart, des enseignants-chercheurs (l'équipe comporte également quelques entrepreneurs et consultants). Ce sont donc aussi des praticiens, en l'occurrence de l'enseignement et de la recherche. Le transfert des connaissances de la recherche vers l'enseignement a été réalisé grâce à des recherches-actions pédagogiques (exemples : Krémer et Verstraete, 2014 ; Krémer, Jouison-Lafitte et Verstraete, 2015). Mais elles ont été plus nombreuses dans les faits, l'équipe acceptant d'appliquer les protocoles rigoureux y compris sur des terrains se prêtant peu à la valorisation scientifique, mais à la valorisation pédagogique évidente.

Les méthodes ainsi mises au point ont permis de sensibiliser à l'entrepreneuriat plus de 10000 étudiants depuis 2008. L’approche avec le terrain a été facilitée par la création d'ECA (Entrepreneuriat Campus Aquitaine), labellisé Pôle de l'entrepreneuriat étudiant par le MENESR (ministère de l'Éducation nationale, de l'Enseignement supérieur et de la Recherche) en 2010 et PEPITE (pôles étudiants pour l'innovation, le transfert et l'entrepreneuriat) en 2014. À l'occasion de la création du nouveau diplôme "étudiant-entrepreneur » par le MENESR, la Charte de labellisation nationale afférente référence GRP Lab comme l'un des trois centres de ressources numériques mobilisables par les porteurs de projet. Le message à retenir est ici de surveiller les appels à projets, car ils fournissent l'occasion de construire une offre structurée, de mobiliser les acteurs locaux, notamment institutionnels (toujours sensibles à une reconnaissance nationale).

Suite à une présentation de GRP Lab lors de la semaine du management de la FNEGE en $2014^{11}$ et lors de la journée nationale des PEPITE précédant le congrès de l'Académie de

10 Ce paragraphe est tiré d'une publication précédente.

11 Également l'occasion de nouer des relations avec BPI France Le Lab. 
l'entrepreneuriat et de l'innovation en 2015 à Nantes, les demandes de formation à la maitrise du modèle GRP et aux outils de GRP Lab se multiplient.

Outre le terrain de la pédagogie et celui de l'accompagnement de porteurs de projets de création d'entreprise, les chercheurs de l'équipe souhaitaient apprécier la pertinence du modèle auprès de dirigeants d'entreprises établies (si possible entrepreneurs). Il s'avère que des relations d'un membre de l'équipe, Jean Dondi, avec la FFBG (Fédération française du bâtiment de Gironde) ont permis de relever un problème que son Président a exprimé de la façon suivante : "face à la concurrence d'entreprises en provenance d'autres territoires, les entreprises du bâtiment de Gironde ${ }^{12}$ devraient gagner en taille pour répondre de façon plus convaincante aux appels d'offre des marchés publics ». La croisée des deux problèmes (celui des chercheurs et celui des représentants de la FFBG) a ouvert un terrain de recherche constitué de 5 jeunes dirigeants (deux femmes, trois hommes) ayant récemment repris l'entreprise familiale pour quatre d'entre eux (le cinquième est un repreneur personne physique externe). Bien que désireux d'entreprendre sur la base de l'entité reprise, ces jeunes entrepreneurs étaient submergés par leur quotidien et ne prenaient pas le temps de la réflexion stratégique. Il a fallu également constater que les grands modèles stratégiques, pourtant tant enseignés dans les universités et les écoles du monde entier, leur étaient soit inconnus, soit inaccessibles. La recherche-action déployée a permis de constater que le concept de BM était compris par ces dirigeants qui voyaient l'intérêt de le mobiliser. Outre ces intéressants et motivants résultats, j’ai sollicité 10 chercheurs dans ce travail. Un binôme de chercheurs pour chaque entrepreneur. L'idée était de mobiliser le cœur de l'équipe à construire ensemble le protocole de collecte et d'analyse des données visant à mettre au jour les BM des entreprises étudiées. Cette démarche collective avait deux objectifs : souder les membres d'une équipe de recherche et s'assurer de l'appropriation du modèle GRP par chacun d'entre eux. Le message à retenir est ici qu'il faut provoquer des recherches collectives conduisant les chercheurs de l'équipe à travailler ensemble pour créer des liens forts entre eux. Ces liens ont également été renforcés, d'une part, par les retours d'un terrain particulièrement enthousiaste à l'issue du travail effectué et, d'autre part, par la valorisation scientifique du travail (communications et articles). À ce titre, toujours dans le même esprit, la publication a été écrite à 11. Tous les participants sont en effet signataires du papier publié dans la Revue de l'Entrepreneuriat (Verstraete, JouisonLaffitte, Krémer, Hlady, Boncler, Boucher, Bousquet, Dondi, Meiar, Papin et Scipion, 2012).

Trois autres terrains de recherche peuvent être ici décrits comme particulièrement représentatifs des travaux de l'équipe et de ses rapprochements avec des terrains tirant des apprentissages de ces rapports « Université/Entreprise ».

Le premier concerne le monde du vin ${ }^{13}$. Mais avant de l'évoquer, rappelons le mythe du garage dans le domaine de l'entrepreneuriat.

12 La Gironde est un département administratif de la région Aquitaine dans le Sud-Ouest de la France.

13 Axe de recherche bénéficiant de l'expertise de Gérard Néraudau, entrepreneur ayant dirigé des entreprises dans ce secteur durant une vingtaine d'années et du recrutement, en 2013, d'une compétence de recherche dans ce domaine avec Franck Duquesnoy. 
Audia et Rider $(2005)^{14}$ ont produit un article rappelant une célèbre croyance populaire américaine, celle du garage dans lequel William Hewlett et David Packard ont conçu un oscillateur les ayant conduit à la création de la firme que l'on connaît. Ce garage a été consacré par une plaque le déclarant "Birthplace of Silicon Valley». Il se situe au 367 Addison Avenue à Palo Alto, non loin de l'Université Stanford. Les auteurs expliquent que le garage est un symbole de l'image américaine de l'entrepreneuriat, de la liberté d'entreprendre, d'être son propre patron, etc. Cisco, Intel, Apple (quoique récemment Steve Wozniak démentait le mythe du garage pour Apple) sont quelques autres emblèmes de ce rêve américain.

En France, le mythe du garage s'est également manifesté, notamment dans le domaine du vin. La réputation des vins français est mondiale, ancrée dans une histoire millénaire et s'exprime dans un secteur insuffisamment mis en avant par le politique alors que la contribution à l'économie française est patente. Si l'ampleur singulière des affaires qui y sont associées n'est pas comparable à l'exemple précédent (HP), et parce que l'équipe est intéressée par les PME, quelques cas remarquables de ce secteur d'activité peuvent attirer l'attention, notamment celui qui est à l'origine des «vins de garage » : Jean-Luc Thunevin. Avec sa compagne, Murielle Andraud, ils ont bousculé l'establishment des vins bordelais (on pourrait dire qu'ils ont bousculé les conventions). Leur cru fétiche, Château Valandraud, est désormais mondialement connu et une bouteille du millésime 2009 dépasse les 300 dollars en prix de vente et a été promu Premier Grand Cru Classé lors du classement 2012 des vins de Saint-Émilion. Pour des autodidactes, la réussite est exceptionnelle.

Notre désir d'apprendre sur cet exemple remarquable nous a conduit à recourir à une étude de cas. Le fait que Jean-Luc Thunevin soit l'initiateur du mouvement garagiste n'est pas étranger à ce choix de cas, son classement récent, alors que la recherche était en cours, ajoute évidemment à l'intérêt qu'il suscite. Dans une vidéo, Jean-Luc Thunevin explique pourquoi avoir accepté de recevoir l'équipe et les enseignements qu'il en a tirés (https://vimeo.com/ grplab). Un consultant réputé de la place bordelaise a aussi pris part à l'expérimentation et livre ses impressions.

Le message à retenir est double. Premièrement, ce genre de cas nous apprend énormément sur les contextes. Ces cas sont remarquables et le chercheur est alors totalement libéré de la quête de l'apport théorique attendu par les revues. Deuxièmement, c'est avec grand regret qu'il faut constater que cette forme d'étude de cas séduit peu les revues en sciences de gestion.

Le deuxième terrain concerne l'axe BM et pédagogie de l'entrepreneuriat piloté par Florence Krémer, et plus précisément la relation avec le rectorat de l'Académie de Bordeaux et une institution nommée Cap Sciences dédiée à la diffusion de la culture scientifique. Les outils de GRP Lab vont être, dès 2016, utilisés dans quelques lycées de l'enseignement secondaire pour une expérimentation de sensibilisation à l'entrepreneuriat selon un protocole nouveau s'appuyant sur un dispositif existant. Deux nouveaux outils, toujours gratuits, prendront place sur GRP Lab en janvier 2016. Ils répondent aux besoins de la cible visée, les résultats du travail de médiation de Cap Sciences ayant été intégrés au cahier des charges informatique. Une recherche-action encadre l'ensemble et des représentants des institutions concernées

14 Audia, P.G. et Rider, C.I. (2005). A garage and an idea : what more entrepreneur need ? California Management Review, 48(1). 
participent désormais aux réunions de l'équipe de recherche, toujours pour créer davantage de liens entre des acteurs travaillant tellement mieux ensemble lorsqu'ils se connaissent ${ }^{15}$.

Un troisième terrain concerne l'entrepreneuriat social auquel s'intéressent particulièrement deux membres de l'équipe, Martine Hlady-Rispal et Jérôme Boncler (une thèse a démarré cette année sur ce thème). La Chaire Entrepreneuriat, hébergée par la fondation Université de Bordeaux, a été créée pour procurer une source de financement à GRP Lab. Son premier donateur, la Banque Populaire Aquitaine Centre Atlantique, a parfaitement intégré la nécessité d'alimenter GRP Lab des produits de la recherche. La fondation est en relation avec d'autres donateurs impliqués dans d'autres chaires. Deux donateurs ont été séduits par l'utilité pratique des recherches de l'équipe valorisée par GRP Lab. Intéressés par l'entrepreneuriat social, ces deux donateurs ont versé un don permettant à l'équipe de développer son axe de recherche : $\mathrm{BM}$ et entrepreneuriat social. Le message à retenir est que l'utilité pratique d'un travail scientifique permet d'obtenir des fonds pour la recherche. Certes, ici le travail pourra déboucher sur un usage du modèle GRP pour les terrains de l'entrepreneuriat social, mais les contacts en cours dévoilent d'ores et déjà le potentiel de GRP Lab pour effectuer des levées de fonds pour la recherche (sans promesse de transfert). Il s'agit d'établir un jeu gagnant-gagnant.

D'autres recherches sont en cours et se répartissent dans 4 axes : BM et pédagogie de l'entrepreneuriat, $\mathrm{BM}$ et accompagnement, $\mathrm{BM}$ et entrepreneuriat social, $\mathrm{BM}$ dans le secteur du vin.

\section{CONCLUSION}

Certes, l'activité scientifique peut se concevoir en autonomie des milieux susceptibles d'utiliser les résultats des travaux scientifiques (Kuhn, 1983). Elle doit pouvoir se conduire indépendamment des attentes de ces milieux. Il ne faudrait pas que ceux-ci imposent au chercheur la question à poser dans ses recherches (Stengers, 1995) ${ }^{16}$ et que la création de valeur économique prenne le pas sur la production de connaissances scientifiques. Ces deux aspects ne sont néanmoins pas antinomiques. Callon et Law $(1998)^{17}$ montrent que la croissance de l'industrie des matières plastiques s'explique par les efforts en recherche fondamentale.

Outre l'idée que je me fais du rôle du chercheur en sciences de gestion (Verstraete, 2007) ${ }^{18}$, l'équipe tout entière a fait un choix qu'elle assume. Nos pratiques de la recherche témoignent de la porosité d'une hypothétique frontière entre le monde de la recherche et celui des pra-

15 Tous les membres de l'équipe s'intéressent à l'axe de recherche en pédagogie de l'entrepreneuriat qui bénéficie également de liens anciens avec des entrepreneurs offrant du temps aux étudiants, notamment Fabrice Scipion.

16 Stengers, I. (1995). L'invention des Sciences modernes. Paris, Flammarion.

17 Callon, M. et Law, J. (1998). La protohistoire d'un laboratoire. Dans M. Callon (dir.), La Science et ses réseaux - Genèse et circulation des faits scientifiques. Paris, Éditions La Découverte.

18 Verstraete, T. (2007). À la recherche des sciences de gestion. Revue française de gestion, 34, (178179). 
tiques étudiées. Cette frontière est entretenue par des cadres opératoires désincarnés et par trop peu d'efforts de rencontres de ces deux mondes s'ignorant trop souvent.

Du côté académique, la production reste, à mon sens, principalement motivée par une équipe de chercheurs constituée autour d'une représentation partagée de son agenda scientifique. Je ne sais plus qui a initialement utilisé la métaphore du troupeau de chats pour évoquer la difficulté à diriger une équipe de chercheurs (qui plus est à une période où, heureusement, le mandarinat perd du terrain). À cet effet, nous avons commencé par concevoir notre propre BM, notamment pour partager notre représentation, pour aussi pouvoir y revenir ou pour l'amender afin de garder la cohésion des différentes parties prenantes à ce qu'on pourrait quasiment qualifier de notre projet d'entreprendre. Est-ce impertinent de croire qu'à nouveau, et trop souvent, les chercheurs en sciences de gestion, en entrepreneuriat et/ou en PME, ne s'appliquent pas les modèles qu'ils enseignent ? On n'est pas pragmatique quand on le croit, mais quand on le fait.

\section{RÉFÉRENCES}

BOUSQUET, F. (2014). L'influence du lien personnel entre l'entrepreneur et le territoire sur l'ancrage territorial des PME (Thèse de doctorat en sciences de gestion). Bordeaux, Université de Bordeaux.

Fouda, N. (2014). Dynamisme entrepreneurial des femmes camerounaises. Études de cas de développement d'entreprise (Thèse de doctorat en sciences de gestion). Bordeaux, Université de Bordeaux.

Hlady-Rispal, M. (2002). La méthode des cas - Application à la recherche en gestion. Bruxelles, De Boeck Université.

Jouison, E. (2008). L'opérationnalité du business model en contexte de création d'entreprise : rechercheaction sur le terrain des porteurs de projet de création d'entreprise (Thèse de doctorat en sciences de gestion). Bordeaux, Université Montesquieu Bordeaux IV.

Jouison-Laffitte, E. (2009). La recherche action : oubliée de la recherche dans le domaine de l'entrepreneuriat. Revue de l'Entrepreneuriat, 8(1), 1-35.

Jouison, E. et Verstraete, T. (2008). BM et création d'entreprise. Revue française de gestion, 181(1), 175-197.

Krémer, F., Jouison-Laffitte, E. et Verstraete, T. (2015). Learning and teaching the business model : the contribution of a specific and dedicated web application. $3^{e}$ Conference - ECSB Entrepreneurship Education Conference. Lunebourg, Allemagne.

Krémer, F. et Verstraete, T. (2014). La carte mentale pour favoriser l'apprentissage du business model et susciter la créativité des apprenants. Revue internationale PME, 27(1), 65-98.

Masamba, V. (2013). La contribution de la relation d'accompagnement pour l'apprentissage de la convention d'affaires inhérente à l'organisation impulsée: une recherche-action au sein de l'incubateur İ F entrepreneuriat en République démocratique du Congo (Thèse de doctorat en sciences de gestion). Bordeaux, Université de Bordeaux.

MeIAR, A. (2015). Le business model de l'entreprise transmise : comparaison de la représentation du repreneur avec celle du cédant pour contribuer à réduire le risque de faux pas (Thèse de doctorat en sciences de gestion). Bordeaux, Université de Bordeaux. 
SERVANTIE, V. (2010). L'internationalisation précoce et rapide des entreprises, une lecture par le business model (Thèse de doctorat en sciences de gestion). Bordeaux, Université Montesquieu Bordeaux IV.

Verstraete, T. et Jouison-Laffitte, E. (2009). Business model pour entreprendre - Le modèle GRP : théorie et pratique. Bruxelles, De Boeck Université.

Verstraete, T. et Jouison-Laffitte, E. (2011a). A conventionalist theory of the business model in the context of business creation for understanding organizational impetus. Management International, 15(2), 109-124.

Verstraete, T. et Jouison-Laffitte, E. (2011b). A Business model for entrepreneurship. Cheltenham, Edward Elgar Publishing.

Verstraete, T., Jouison-laffitte, E., Krémer, F., Hlady, M., Boncler, J., Boucher, T., Bousquet, F., Dondi, J., Meiar, A., Papin, C. et Scipion, F. (2012). Recherche-action pour apprécier l'utilité du concept de business model pour les jeunes dirigeants d'entreprise du bâtiment. Revue de l'Entrepreneuriat, 11(4), 67-94. 